\title{
Web-Based Intervention Preferences and Physical Activity Motivation of People with Depressive Symptoms
}

\author{
Stephanie Alley*, Martina Andersson ${ }^{\dagger}$, Nicola Burton ${ }^{\ddagger}$, Corneel Vandelanotte*, Mitch \\ Duncan, Brenda Happellll, Camille Short? and Amanda Rebar*
}

\begin{abstract}
Physical activity is an effective method for managing depressive symptoms, and web-based interventions may be an effective tool for promoting physical activity within this population. People with depressive symptoms may experience condition-specific facilitators and barriers to be considered when developing physical activity interventions. This study provides insight into web-based physical activity intervention preferences and physical activity motivation of people with depressive symptoms. This study was part of a larger trial of a web-based physical activity intervention for people with depressive symptoms. Thematic analysis of participants' $(N=20)$ written responses to open-ended survey questions was conducted. Preferences for web-based intervention features included social forums, self-monitoring, suggestions for types of physical activity in different circumstances and information targeted to people with depressive symptoms. When discussing physical activity motivation instrumental beliefs (i.e., beliefs of the benefits and cost of physical activity) and controllability (i.e., whether the behaviour is under their control) were discussed. Additionally, people reported condition-specific barriers of physical activity such as depressive symptoms of apathy, worthlessness, and pain. Further research is needed to test the effectiveness of web-based physical activity interventions targeting people with depressive symptoms, which incorporates these findings.
\end{abstract}

Keywords: mood disorders; exercise; intentions; e-health

Depression is highly prevalent and is associated with significant societal and individual costs. Worldwide, more than 350 million people have depression and it is the leading cause of disability (World Health Organization, 2014). Depression can result in profound impairments in quality of life and wellbeing (Rapaport, Clary, Fayyad, \& Endicott, 2005; Rhebergen et al., 2010) and, as a result of pervasive depressive symptoms, people typically lose interest in hobbies, withdraw from activities, and experience impairments in social and occupational functioning (American Psychiatric Association, 2013).

Some relief of depressive symptoms can result from conventional treatment options including pharmacotherapy, psychotherapy, or the combination of these therapies; however, there are several limitations to these treatment options such as the cost and accessibility of services (Gabriel, \& Violato, 2010), anticipated

\footnotetext{
* Central Queensland University, AU

+ Queensland Government, AU

₹ The University of Queensland, AU

§ The University of Newcastle, AU

॥ University of Canberra, AU

" University of Adelaide, AU

Corresponding author: Stephanie Alley (s.alley@cqu.edu.au)
}

stigmatisation (Acosta, Rodríguez, \& Cabrera, 2013), and adverse side-effects like insomnia, sedation and neurological or sexual dysfunction (American Psychiatric Association, 2013). As a result of these limitations, a large number of people with depression are not receiving help for symptoms. Nearly half (45\%) of Australians in need of mental health treatment are not receiving treatment (Kohn, Saxena, Levav, \& Saraceno, 2004). As such, there is an important need to explore other easily accessible treatments to reduce depressive symptoms.

\section{Physical activity as a treatment option for depressive symptoms}

Physical activity has been shown to significantly alleviate depressive symptoms (Cooney et al., 2013; A. L. Rebar et al., 2015). Some evidence suggests that the antidepressant benefits of physical activity may have the same magnitude of effect, or even larger, than that of pharmacological or psychotherapy treatments (Cooney et al., 2013; Rethorst, Wipfli, \& Landers, 2009). The antidepressant effects of physical activity have been explained partly by enhanced regulation of hippocampal neurogenesis, including increased serotonin (Ernst, Olson, Pinel, Lam, \& Christie, 2006) and noradrenaline (Dishman, 1997). Physical activity also has antidepressant effects via psychological mechanisms such as enhanced self-esteem (Ossip-Klein, 
Doyne, Bowman, Osborn, \& Neimeyer, 1989), self-efficacy and coping ability (Pickett, Yardley, \& Kendrick, 2012).

\section{Web-Based Physical Activity Interventions}

Web-based physicalactivityinterventions havethepotential to offer participants advice at any time and place for a lower cost than face-to-face physical activity interventions (Vandelanotte, Spathonis, Eakin, \& Owen, 2007). Webbased physical activity interventions also remove stigma of traditional treatments for depressive symptoms and delivers the treatment in a non-confrontational manner (Tate, Finkelstein, Khavjou, \& Gustafson, 2009). Research has shown that web-based physical activity interventions can increase physical activity behaviour (Davies, Spence, Vandelanotte, Caperchione, \& Mummery, 2012), however we do not know the perceptions and preferences of webbased interventions in people with depressive symptoms. Such knowledge is needed to inform the development of future web-based physical activity interventions effective at engaging this population group.

\section{Understanding physical activity behaviour}

Understanding the physical activity behaviour of people with depressive symptoms is important for the development of interventions targeting this population group. Behaviour theories, such as the theory of planned behaviour, are used to explain physical activity behaviour of individuals and guide intervention content. Webbased physical activity interventions based on the theory of planned behaviour are more effective at changing behaviour (Ajzen, 1988; Webb, Joseph, Yardley, \& Michie, 2010). Interventions based on the theory of planned behaviour target individuals' behavioural intentions (i.e., people's plans to engage in the behaviour) by addressing attitudes (i.e., positive or negative evaluations), subjective norms (i.e., perceived social pressure and support) and perceived behavioural control (i.e., perceived ability to execute the behaviour; opportunities; resources) (Ajzen, 1988). Attitudes can be classified as either instrumental (i.e., beliefs of the benefits and cost of behaviour as being beneficial or harmful) or affective (i.e., emotionbased evaluations of the behaviour as being enjoyable or unenjoyable); subjective norm includes an injunctive (i.e., whether one believes their social network wants them to perform the behaviour) and a descriptive component (i.e., whether one's social network performs the behaviour); and perceived behavioural control has two subcomponents of self-efficacy (i.e., perceived ease/difficulty of performing the behaviour and confidence) and controllability (i.e., personal control over behaviour, appraisal of whether one's self has control over the behaviour).

The emotional, cognitive, and motivational manifestations of depressive symptoms may affect attitudes, social norms and perceived behavioural control for physical activity (Rhebergen et al., 2010). The main physical activity barriers for people with depressive symptoms include lack of family support, negative physical activity experiences, external factors (e.g., lack of time and money) and depressive symptoms (Azar, Salmon, \& Cleland, 2010; Rogerson, Murphy, Bird, \& Morris, 2012). Physical activity motivators identified for people with depressive symptoms include weight control, health benefits and seeing results (Azar et al., 2010; Rogerson et al., 2012). Whilst this research identifies key physical activity attitudes in this population group, further research is needed to investigate all of the theory of planned behaviour constructs for physical activity in people with depressive symptoms to inform the content of future interventions targeting this population group.

\section{The Present Study}

Given the scarcity of previous evidence, qualitative research is an essential first step to gain insight into the perceptions of people with depressive symptoms regarding web-based physical activity intervention preferences and physical activity motivation. This data will help to inform the delivery (e.g., web-based), components (e.g., forums, self-monitoring) and content (i.e., addressing attitudes specific to the target group) of physical activity interventions for people with depressive symptoms. The present study aimed to examine web-based intervention preferences within people with depressive symptoms and physical activity motivation using the framework of the theory of planned behaviour.

\section{Method \\ Participants}

This study recruited adults (18 years or older) who previously participated in a larger trial evaluating the effects of web-based physical activity interventions on depressive symptoms and physical activity (Amanda L. Rebar et al., 2016). All study procedures were approved by the relevant Human Research Ethics Committee (approval number: H13/08-147). For the larger trial 514 participants were recruited via Facebook and Google advertising targeting people with low mood (Amanda L. Rebar et al., 2016). The 2-arm randomised trial compared the effectiveness of two web-based interventions for increasing physical activity and reducing depressive symptoms. Participants were randomised to receive either a web-based physical activity intervention program with interactive features or a webbased physical activity intervention with personalised advice. Physical activity significantly increased and depression significantly decreased from baseline to one month and three months and no between group differences on physical activity or depression were found (Amanda L. Rebar et al., 2016). For the current study participants in the personalised advice arm of the trial who reported depressive symptoms were recruited (based on a score $>16$ on the CES-D-r validated measure of depression (Van Dam, \& Earleywine, 2011). E-mail invitations were sent out to 50 potential participants, a size estimated a priori to be sufficient to achieve saturation with the anticipated response rates between 25 to 50\% (Baker, \& Edwards, 2006).

\section{Procedure and Materials}

Participants' demographics, BMI and physical activity behaviour was already collected through the larger trial. Demographics included age, sex, education (<year 12, >year 12) and household income $(<52,000$ AUD, 
$>52,000$ AUD). BMI was calculated from self-reported height and weight, and physical activity was assessed using the Active Australia Survey (Australian Institute of Health and Welfare, 2003) which has previously been shown to have acceptable reliability and validity (Australian Institute of Health and Welfare, 2003; Fjeldsoe, Winkler, Marshall, Eakin, \& Reeves, 2013). The Active Australia survey measures total time walking, participating in vigorous physical activity (e.g., jogging, cycling, aerobics, competitive tennis) and participating in moderate physical activity (e.g., gentle swimming, social tennis, golf) in the past week. Minutes of physical activity per week were calculated as the sum of the time spent walking, time in moderate activity and time in vigorous activity (weighted by two to account for the extra energy expenditure output) as per manual instructions (Australian Institute of Health and Welfare, 2003).

The invitation e-mails included information about the study and the open-response questions. The questions were formulated to explore factors associated with participants' physical activity motivation, their knowledge of physical activity, their knowledge of other treatments to improve mood and perceptions and preferences of webbased physical activity interventions. The questions on physical activity motivation were, "What motivates you to be physically active?," "What stops you from being physically active?," "What benefits do you experience from being physically active?," "What do you think of being physically active as a way to feel better emotionally? Does it work?" and "How active do you think you need to be to improve your mood and reduce negative feelings?," Participants were also asked, "What are some methods that you are aware of to help improve mood and reduce feelings of sadness or irritability and how well do you think they work?," "Have you had any experiences with using various treatments to improve your mood?," "What are limitations of the treatments that you may have tried?" and "Has anything stopped you from trying other treatments to feel better emotionally?" Questions about physical activity web-based interventions were then asked. First it was explained to participants that "We are interested in developing a website to promote physical activity for people who want to improve their mood and reduce feelings like sadness, irritability and fatigue." Participants were then asked "Would you use a site like this? Why or why not?," "What features would you like such a website to have?," "Do you think a website like this could help you get motivated to be physically active? Why or why not?" and "How could a physical activity website specifically be designed to help people who have problems with low moods become more active?" Willing participants provided their responses to these questions by return email.

\section{Analysis}

Data were analysed using the thematic analysis procedure as outlined by Braun and Clarke (2006). The procedure involves 9 steps including (1) familiarising yourself with your data; (2) generating initial codes; (3) searching for themes; (4) reviewing themes; (5) defining and naming themes and (6) producing the report. For the data on web-based physical activity interventions, participant themes were identified in the data. For the data on factors affecting physical activity motivation, a theoretical approach was used. Here the data were analysed based on themes relating to, and beyond, the theory of planned behaviour framework. Specifically, the surveys were read and reviewed by MA to generate initial codes relating to, and beyond, the theory of planned behaviour (i.e., instrumental and affective attitudes; injunctive and descriptive subjective norm; and the subcomponents of perceived behavioural control, self-efficacy and controllability). Each response was then coded by both MA and ALR. Any inconsistencies were resolved by discussion between MA and ALR. As additional themes were revealed, participants' responses were reanalysed reiteratively.

\section{Results}

Responses were received from twenty participants (40\% response rate) and then analysed. Both authors involved in data analysis (MA \& ALR) considered there was saturation and therefore decided not to invite additional people to participate in the study. Descriptive characteristics of the study sample is presented in Table $\mathbf{1}$. The sample was mostly female $(n=14,70 \%)$ and the mean age was $45 \pm 18$. This was similar to the larger trial which was $70 \%$ female with an average age of $46 \pm 15$. Participants reported a mean sore of $29.5(\mathrm{SD}=8.9)$ on the CES-D-r scale. The large standard deviations for age, BMI, physical activity and depressive symptoms highlight a large diversity in participants.

\section{Web-based Intervention Preferences}

Consideration for depressive symptoms. Many participants reported that it was important for physical activity web-based programs to account for depressive symptoms, specifically the symptom of low mood. They suggested beneficial features could either teach strategies or suggest methods of improving low mood: "The recognition that exercise is very difficult for people with mood issues would do a lot more to help than any GP has ever done (Female, 42)," "If a person is feeling low, depressed etc. the problem is will that person at that moment of need actually check out a website (Male, 79)." These participants had a broad range of frequencies of depressive symptoms.

Social networking. Many participants desired a webbased physical activity program to have features that encouraged social networking and increased social support from likeminded people: "A website with community features and options for people to share their stories with each other from similar circumstances can work to push each other (Female, 20)," "Also a site where people could encourage others and suggest different forms of physical activity that worked for them may be beneficial (Female, 37)." The participants that desired social networking tended to be females under 50 years of age.

Self-monitoring. Many participants reported that features which allowed users to keep track record of their achievement would encourage further use of the website and create an opportunity to visualise benefits of regular physical activity: "Being able to see how much exercise I've 
Table 1: Descriptive Statistics of Participants' Demographic Characteristics.

\begin{tabular}{|c|c|c|}
\hline & $M$ & $S D$ \\
\hline Age (years) & 45.2 & 18.18 \\
\hline BMI & 30.43 & 8.91 \\
\hline Physical activity; minutes/week & 139.33 & 176.57 \\
\hline \multirow[t]{2}{*}{ Depression symptom frequency (CESD-r score) } & 29.5 & 8.86 \\
\hline & $\mathbf{n}$ & $\%$ \\
\hline \multicolumn{3}{|l|}{ Gender } \\
\hline Female & 14 & 70 \\
\hline Male & 6 & 30 \\
\hline \multicolumn{3}{|l|}{ Education } \\
\hline <year 12 & 9 & 45 \\
\hline >year 12 & 11 & 55 \\
\hline \multicolumn{3}{|l|}{ Household Income } \\
\hline$<$ AUD\$52000 pa & 6 & 30 \\
\hline >AUD\$52000 pa & 9 & 45 \\
\hline Not reported & 5 & 25 \\
\hline
\end{tabular}

Note. CESD-r: Center for Epidemiologic Studies Depression Scale -revised on which scores $>16$ represent clinical depressive symptoms.

been doing over the last month or so really motivates me not to skip a day (Female, 19).

Suggestions for physical activity in different circumstances. Some participants reported that a webbased program with suggestions about a variety of physical activities may allow for people to be active while having a busy lifestyle or experiencing physical discomfort: "Perhaps it could have quick ideas for ways to be active in different circumstances. Like, how can I be active for 10 mins if I'm busy, or I travel a bit so maybe what can you do when you are not at home (Female, 49)." The participants that were interested in physical activity suggestions tended to be female.

\section{Factors affecting physical activity motivation}

Consideration for depressive symptoms. A theme sepa rate to the theory of planned behaviour emerged relating to the participants' attempts to be physical active while experiencing depressive symptoms. Some participants indicated that depressive symptoms of apathy, worthlessness, and pain created a significant barrier to being physical active: "Generally it's depression. A feeling of pain and tension, lack of sleep, worthlessness. Then I don't want to do anything (Male 47)," "Sometimes apathy brought on by mild depressive episodes (Female, 37)," "Mental condition that leads to procrastination through fear of failure (Male, 59)." These participants had a broad range of frequencies of depressive symptoms.

Perceived behavioural control - controllability. Many participants gave responses about physical activity motivation related to controllability (i.e., feelings of control over resources and opportunities for being physically active). Each participant mentioned lack of time as a barrier to being physically active, and other barriers were reported, including cost of gym membership, lack of energy due to work, sedentary work, and family commitments. Such responses include, "Lack of time and sedentary job (Female, 54)," "Finding time to do activity usually stops me (Female, 49)," "The major restriction on being active, such as joining a sporting team, is largely down to external pressures like, time, cost, distance etc. (Male, 57)." One respondent reported that that having time set aside first thing in the morning increased likelihood of engaging in physical activity.

Perceived behavioural control - self-efficacy. Some participants' responses highlighted self-efficacy (i.e., perceptions of ability to perform physical activity) as a factor affecting physical activity. Some indicated that physical activity induced feelings of physical activity as being too difficult, or that they lacked the confidence to pursue an active lifestyle. Such responses included, "Fear of actually trying and not being good enough, finding small things too hard and being noticed. It's hard to try and utilise competitiveness as a motivational drive when you don't feel good enough to compete and won't start out of shame (Female, 20)," and "I liked dance as a kid, and joined a few classes over the years but I don't have the confidence to join a dance class these days (Female, 42)." Other Participants indicated that they felt like physical activity increased their sense of competence and facilitated more active behaviours: "The thought of being able to run, or dance, or do others physical things, and actually be able to do it and enjoy it, makes me want to get fit (Female, 23)," "I enjoy doing things that I couldn't before (Female, 49)." The participants whose responses reflected self-efficacy (both positive and negative) tended to be females under 50 years of age.

Attitude - instrumental beliefs. The majority of participants emphasised instrumental beliefs as being a source of motivation for physical activity (e.g., benefits). Fourteen participants indicated that fitness goals, healthy lifestyle and weight control motivated them to engage in physical activity, and some participants also said that they were motivated to be physically active because it reduced physical pain, discomfort, and stress. Responses included, "I am motivated by wanting to be physically stronger, able to run father without visibly being overworked, and I'd like to be leaner as appropriate to my body shape (Female, 20)," "To lose weight, have a clearer head space, reduce fibromyalgia 
issues - reducing back pain, improve sleep and reduce stress and anxiety (Female, 18)," and "Usually what motivates me to be physically active is the idea that exercise will make me better physically. I like to play sport etc so the idea of being faster or stronger is a big motivator (Female, 49)." Whilst both males and females of many ages mentioned instrumental beliefs, it tended to be females who discussed the benefits of physical activity related to appearance.

Some participants' responses demonstrated negative instrumental beliefs that affected their physical activity motivation (e.g., costs of physical activity). This included physical discomfort and that they did not see any benefit from exercise: "When I do attempt to work out, the 'afterburn' of a workout generally lasts for several days, which makes it hard to want to pick up a 3 times a week routine (Female, 20)," "Physical discomfort, and the fact that my body very stubbornly holds on to weight and exercise doesn't help weight loss unless I work really hard which is unsustainable for me because I don't enjoy it (Female, 42)."

Attitude - affective evaluations. Some participants had responses indicative of affective attitudes (i.e., evaluations of physical activity as fun and enjoyable). Some participants indicated that they enjoyed sports and physical activity or found it peaceful: "I love being outside, particularly cycling along the banks of the river. It's peaceful, scenic, and it allows a kind of meditation (Female, 64)," "I enjoy playing sports and games casually (Male, 57)." In some cases, affective attitudes were described as barriers to physical activity: "I have struggled with exercise all my adult life. I hate it (Female, 42)," "The biggest thing that stops me from being physically active is that I really, really don't feel like it. The thought of deliberately going and doing something for no purpose other than the sake of doing it. That is no fun (Female, 23)." Females under 50 were more likely to describe affective attitudes as barriers to physical activity.

Subjective norm - injunctive factors. There were a few participants who indicated injunctive factors (i.e., experienced social expectations to engage in physical activity) to be an important aspect of physical activity motivation. Participants reported injunctive factors as both a barrier: "Because in team sport there is still pressure to perform etc (Female, 49)," as well as a facilitator: "I have a friend that I confided in when I felt like I was bored with my life and needed a hobby, and he suggested working out. That's my main source of motivation, to prove to him that I can stick to a workout routine and see it through (Female, 19)."

\section{Discussion}

The findings revealed intervention preferences for people with depressive symptoms, and factors affecting physical activity motivation in people with depressive symptoms. These results are important for informing the development of future physical activity interventions which meet the specific needs of people with depressive symptoms.

Web-based physical activity intervention preferences Interactive features which target social support (e.g., social forums as a medium for connecting to other people, having advice from like-minded people about getting active) were the most frequently suggested features for webbased physical activity programs. This may be that con- necting with others online is not as confrontational and gives the user more control of the interaction when compared to face-to-face meetings (Breuer, \& Barker, 2015; Evans, Donelle, \& Hume-Loveland, 2012). Fitting with past research on health related social media use, it was younger and middle aged females who suggested social support features (Thackeray, Crookston, \& West, 2013). Therefore, we do not know if older females or males would be interested in social support through a web-based intervention.

Participants also reported preferences for web-based program features that self-monitor physical activity. These findings are in line with previous meta-analytic findings that self-monitoring is an engaging and effective means for promoting physical activity in the general population (Michie, Abraham, Whittington, \& McAteer, 2009). In addition, the participants indicated that a web-based program should include suggestions for unique ways to be physically active and alternative physical activities for people with a busy lifestyle. Such features may be important for enhancing perceived behavioural control. Participants with a wide range of depressive symptoms also mentioned that web-based interventions should incorporate features that specifically accommodate for depressive symptoms. For example, it was suggested that promoting 'small goals' could help people overcome feelings of being overwhelmed or discouraged. This is effective in the general population (Gollwitzer, 1999) but may be more important in people with depressive symptoms. Interventions could also provide participants with information about physical activity useful in reducing symptoms of depression such as tension (e.g., tai chi). Such suggestions highlight the importance of tailoring interventions specifically for people with depressive symptoms.

\section{Factors affecting physical activity motivation}

Participants with a wide range of depressive symptoms highlighted that depressive symptoms come with condition-specific motivational factors for physical activity. Separate to the theory of planned behaviour, they indicated that depressive symptoms including pain, tension, feelings of worthlessness, and apathy were barriers to physical activity. This finding, alongside the finding of the need for physical activity interventions to accommodate for depressive symptoms suggests that interventions should address these condition-specific barriers.

Within this sample of people with depressive symptoms, lack of time, cost of gym membership, lack of energy due to work, inactive work environments, and family commitments were frequently reported as barriers of physical activity. This suggests that controllability is an important factor affecting physical activity motivation in this sample. The comments were about lack of control which may be as people with depressive symptoms tend to have an external locus of control where they attribute causes and events to things and people other than themselves (Van Dijk, Dijkshoorn, van Dijk, Cremer, \& Agyemang, 2013). Therefore they may be likely to view physical activity impediments as out of their control and unable to be resolved. People with depressive symptoms might benefit from physical activity intervention tools that provide guidance about how to control the resources and opportunities 
to be active. For example interventions could reinforce that there are many affordable alternatives to the gym (e.g., bike riding, walking, home exercises).

Some participants' responses highlighted low self-efficacy as a barrier to physical activity. This may be as people with depressive symptoms tend to have low self-esteem and confidence (American Psychiatric Association, 2013). Conversely, some participants mentioned that physical activity increases their sense of competence, which may help to improve their overall self-esteem. Interventions could encourage people with depressive symptoms to start with physical activity they feel competent doing (e.g., walking) and, if they have low self-efficacy, avoid competitive sporting environments.

Almost all participants reported instrumental benefits that could motivate them to do physical activity, including meeting fitness goals, healthier lifestyle and weight control. This is important to note, as losing interest in health and appearance can be a symptom of depression. The finding may be due to people with depressive symptoms wanting to improve their symptoms including major alternations in physical health, mood, weight, and sleep (American Psychiatric Association, 2013) through physical activity (Physical Activity Guidelines Advisory Committee, 2008). In line with past research in the general population, the participants who were motivated by appearance tended to be female, whilst both genders mentioned health as a motivating factor (Molanorouzi, Khoo, \& Morris, 2015). Interventions promoting physical activity for people with depressive symptoms should emphasise the physical and mental health benefits of physical activity to both genders, including how it can reduce depressive symptoms.

Some participants, more often female, mentioned affective attitudes as a major barrier to physical activity such as disliking physical activity, particularly strenuous physical activity. These findings are in line with evidence of affective attitudes being a strongly influential element of the theory of planned behaviour in the general population (Downs, \& Hausenblas, 2005; McEachan, Conner, Taylor, \& Lawton, 2011) (Downs, \& Hausenblas, 2005; McEachan et al., 2011), and females' lower participation in strenuous activity (Australian Bureau of Statistics, 2015). People with depressive symptoms might be particularly vulnerable to affective attitudes, given that depressive symptoms include loss of interest and pleasure in activities and hobbies (American Psychiatric Association, 2013). Conversely, some participants mentioned positive effective attitudes towards physical activity such as enjoyment and the love of the outdoors. Physical activity interventions should encourage people, particularly females to engage in physical activity that they enjoy, and if intrinsic enjoyment is low, provide strategies to make the general experience enjoyable e.g., considering aspects of context, location, companionship.

The findings that people with depressive symptoms provided little reference to injunctive norms and no reference to descriptive subjective norms is in line with previous research showing subjective norms have a smaller influence on physical activity in the general population (McEachan et al., 2011). Pressure to perform was mentioned as a barrier to physical activity which may be a reflection of the depressive symptoms of social withdrawal (American Psychiatric Association, 2013). Interventions targeting people with depressive symptoms should therefore give examples of non-competitive types of physical activity (e.g., casual walking or bike riding).

\section{Limitations and Future Directions}

As is the nature of qualitative research on small sample sizes, the findings should not be generalised to all people with depressive symptoms. The sample had diverse characteristics, particularly in terms of age and depressive symptom frequency, which may have contributed to the diversity in responses. This research should be followed up with deductive research with larger samples. Second, the data collection was based on an e-mailed survey of openresponse questions which has the benefit of reduced risk of response bias as a result of anonymity, but it is limited in that it may not have provided as in-depth responses as other methods such as a semi-structured interviews, in which the participants can be prompted to further elaborate on their responses (Sullivan, Gibson, \& Riley, 2012). Although the participants had previously participated in a web-based intervention with personalised advice or interactive features, the questions were about future interventions built specifically for people with depressive symptoms. Therefore, whilst their responses may have been influenced by their experience in that intervention, the responses are useful for the development of interventions specifically for people with depressive symptoms.

This research study contributes valuable insight into the physical activity motivation of people with depressive symptoms and indicates that there are special conditionspecific barriers and facilitators to physical activity that should be accounted for in physical activity interventions targeting this population. Important next steps in this line of research includes future research on the generalisability of these findings across a broader array of people with depressive symptoms and evaluations of web-based physical activity interventions for people with depressive symptoms based on these findings.

\section{Conclusions}

The findings suggest that web-based physical activity interventions for people with depressive symptoms should include features of social forums, self-monitoring, and suggestions for different types of physical activity that can suit their lifestyle and needs. Importantly, the intervention content must address physical activity barriers specific to people with depressive symptoms such as low mood. The findings also suggest that people with depressive symptoms experience barriers they feel are out of their control (e.g., lack of time) and depressive symptoms (e.g apathy and worthlessness) which hinder their participation in physical activity. Many instrumental beliefs and some affective evaluations were listed as both barriers (e.g., discomfort, lack of enjoyment) and facilitators to physical activity (improved health, weight loss, enjoyment). This information is useful for informing future web-based physical activity interventions effective at increasing physical activity in people with symptoms of depression. 


\section{Additional Files}

The additional files for this article can be found as follows:

- Data. Participants' responses to study questions. DOI: https://doi.org/10.5334/hpb.3.s1

- Ethics. Study ethics application and approval. DOI: https://doi.org/10.5334/hpb.3.s2

\section{Peer Review Comments}

Health Psychology Bulletin has blind peer review, which is unblinded upon article acceptance. The editorial history of this article can be downloaded here:

- PR File 1. Peer Review History. DOI: https://doi. org/10.5334/hpb.3.pr1

\section{Competing Interest}

The authors have no competing interests to declare.

\section{References}

Acosta, F., Rodríguez, L., \& Cabrera, B. (2013). Beliefs about depression and its treatments: Associated variables and the influence of beliefs on adherence to treatment. Revista de Psiquiatría Y Salud Mental (English Edition), 6(2), 86-92. DOI: https://doi. org/10.1016/j.rpsm.2012.08.001

Ajzen, I. (1988). Attitudes, personality, and behaviour. Illinois, USA: The Dorsey Press.

American Psychiatric Association. (2013). Diagnostic and statistical manual of mental disorders. Arlington, VA: American Psychiatric Publishing. DOI: https://doi.org/10.1176/appi. books.9780890425596

Australian Bureau of Statistics. (2015). National Health Survey: First Results, 2014-15. Canberra, Australia. Retrieved from: http://www.abs. gov.au/ausstats/abs@.nsf/Lookup/by\%20 Subject/4364.0.55.001 2014-15 Main\%20 Features Exercise 29 .

Australian Institute of Health and Welfare. (2003). The Active Australia Survey. A guide for manual implimentation, analysis and reporting. ISBN: 978-1 74024-258-5. Retrieved from: http://www.aihw.gov. au/publication-detail/?id=6442467449.

Azar, D. B. K., Salmon, J., \& Cleland, V. J. (2010). Physical activity correlates in young women with depressive symptoms: a qualitative study. International Journal of Behavioral Nutrition and Physical Activity, 7(3). DOI: https://doi.org/10.1186/1479-5868-7-3

Baker, S. E., \& Edwards, R. (2006). How many qualitative interviews is enough? Retrieved from: http:// eprints.ncrm.ac.uk/2273.

Braun, V., \& Clarke, V. (2006). Using thematic analysis in psychology. Qualitative Research in Psychology, 3(2), 77-101. DOI: https://doi. org/10.1191/1478088706qp063oa

Breuer, L., \& Barker, C. (2015). Online Support Groups for Depression: Benefits and Barriers. SAGE Open, 5(2). DOI: https://doi.org/10.1177/2158244015574936

Cooney, G. M., Dwan, K., Greig, C. A., Lawlor, D. A., Rimer, J., Waugh, F. R., Mead, G. E. et al.
(2013). Exercise for depression. Cochrane Database Syst Rev, 9, CD004366. DOI: https://doi. org/10.1002/14651858.CD004366.pub6

Davies, C., Spence, J. C., Vandelanotte, C., Caperchione, C. M., \& Mummery, W. K. (2012). Meta-analysis of Internet-delivered interventions to increase physical activity levels. Int $J$ Behav Nutr Phys Act, 9(1), 52. DOI: https://doi. org/10.1186/1479-5868-9-52

Dishman, R. K. (1997). Brain monoamines, exercise, and behavioral stress: Animal models. Medicine \& Science in Sports \& Exercise, 29(1), 63-74. DOI: https:// doi.org/10.1097/00005768-199701000-00010

Downs, D. S., \& Hausenblas, H. A. (2005). The theories of reasoned action and planned behavior applied to exercise: A meta-analytic update. Journal of Physical Activity and Health, 2(1), 76-97. DOI: https://doi. org/10.1123/jpah.2.1.76

Ernst, C., Olson, A. K., Pinel, J. P. J., Lam, R. W., \& Christie, B. R. (2006). Antidepressant effects of exercise: Evidence for an adult-neurogenesis hypothesis? Journal of Psychiatry \& Neuroscience, 31(2), 84-92.

Evans, M., Donelle, L., \& Hume-Loveland, L. (2012). Social support and online postpartum depression discussion groups: a content analysis. Patient Educ Couns, 87(3), 405-410. DOI: https://doi. org/10.1016/j.pec.2011.09.011

Fjeldsoe, B. S., Winkler, E. A., Marshall, A. L., Eakin, E. G., \& Reeves, M. M. (2013). Active adults recall their physical activity differently to less active adults: test-retest reliability and validity of a physical activity survey. Health Promot J Austr, 24(1), 26-31. DOI: https://doi.org/10.1071/HE12912

Gabriel, A., \& Violato, C. (2010). Knowledge of and attitudes towards depression and adherence to treatment: The Antidepressant Adherence Scale (AAS). Journal of Affective Disorders, 126(3), 388-394. DOI: https://doi.org/10.1016/j.jad.2010.07.013

Gollwitzer,P.M.(1999).Implementationintentions:Strong effects of simple plans. Am Psychol, 54(7), 493-503. DOI: https://doi.org/10.1037/0003-066X.54.7.493

Kohn, R., Saxena, S., Levav, I., \& Saraceno, B. (2004). The treatment gap in mental health care. Bull World Health Organ, 82(11), 858-866. DOI: https://doi. org/10.1590/S0042-96862004001100011

McEachan, R. R. C., Conner, M., Taylor, N. J., \& Lawton, R. J. (2011). Prospective prediction of health-related behaviours with the Theory of Planned Behaviour: A meta-analysis. Health Psychol Rev, 5(2), 97-144. DOI: https://doi.org/10.1080/17 437199.2010.521684

Michie, S., Abraham, C., Whittington, C., \& McAteer, J. (2009). Effective techniques in healthy eating and physical activity interventions: A meta-regression. Health Psychol, 28(6), 690-701. DOI: https://doi. org/10.1037/a0016136.supp

Molanorouzi, K., Khoo, S., \& Morris, T. (2015). Motives for adult participation in physical activity: type of activity, age, and gender. BMC Public Health, 15, 66. DOI: https://doi.org/10.1186/s12889-015-1429-7 
Ossip-Klein, D. J., Doyne, E. J., Bowman, E. D., Osborn, K. M., \& Neimeyer, R. A. (1989). Effects of running or weight lifting on self-concept in clinically depressed women. Journal of Consulting and Clinical Psychology, 57(1), 158-161. DOI: https:// doi.org/10.1037//0022-006X.57.1.158

Physical Activity Guidelines Advisory Committee. (2008). Physical activity guidelines advisory committee report. Washington, DC: Department of Health and Human Services.

Pickett, K., Yardley, L., \& Kendrick, T. (2012). Physical activity and depression: A multiple mediation analysis. Mental Health and Physical Activity, 5(2), 125-134. DOI: https://doi.org/10.1016/j. mhpa.2012.10.001

Rapaport, M. H., Clary, C., Fayyad, R., \& Endicott, J. (2005). Quality-of-life impairment in depressive and anxiety disorders. AmJPsychiatry, 162(6), 1171-1178. DOI: https://doi.org/10.1176/appi.ajp.162.6.1171

Rebar, A. L., Boles, C. W., Burton, N., Duncan, M. J., Short, C. E., Happell, B., Vandelanotte, C. et al. (2016). Healthy mind, healthy body: A randomized trial testing the efficacy of a computer-tailored vs. interactive web-based intervention for increasing physical activity and reducing depressive symptoms. Mental Health and Physical Activity, 1129-37. DOI: https://doi.org/10.1016/j.mhpa.2016.08.001

Rebar, A. L., Stanton, R., Geard, D., Short, C., Duncan, M. J., \& Vandelanotte, C. (2015). A meta-meta-analysis of the effect of physical activity on depression and anxiety in non-clinical adult populations. Health Psychol Rev, 9(3), 1-78. DOI: https://doi.org/10.1080/17437199.2015.1022901

Rethorst, C. D., Wipfli, B. M., \& Landers, D. M. (2009). The antidepressive effects of exercise: a meta-analysis of randomized trials. Sports Med, 39(6), 491-511. DOI: https://doi. org/10.2165/00007256-200939060-00004

Rhebergen, D., Beekman, A. T., de Graaf, R., Nolen, W. A., Spijker, J., Hoogendijk, W. J., \& Penninx, B. W. (2010). Trajectories of recovery of social and physical functioning in major depression, dysthymic disorder and double depression: a 3-year follow-up. Journal of Affective Disorders, 124(1), 148-156. DOI: https://doi.org/10.1016/j. jad.2009.10.029

Rogerson, M. C., Murphy, B. M., Bird, S., \& Morris, T. (2012). "I don't have the heart": a qualitative study of barriers to and facilitators of physical activity for people with coronary heart disease and depressive symptoms. International Journal of Behavioral Nutrition and Physical Activity, 9(140). DOI: https://doi. org/10.1186/1479-5868-9-140

Sullivan, C., Gibson, S., \& Riley, S. C. (2012). Doing your qualitative psychology project. London, England: Sage. DOI: https://doi. org/10.4135/9781473914209

Tate, D. F., Finkelstein, E. A., Khavjou, O., \& Gustafson, A. (2009). Cost effectiveness of Internet interventions: Review and recommendations. Ann Behav Med, 38(1), 40-45. DOI: https://doi. org/10.1007/s12160-009-9131-6

Thackeray, R., Crookston, B. T., \& West, J. H. (2013). Correlates of health-related social media use among adults. J Med Internet Res, 15(1), e21. DOI: https:// doi.org/10.2196/jmir.2297

Van Dam, N. T., \& Earleywine, M. (2011). Validation of the Center for Epidemiologic Studies Depression ScaleRevised (CESD-R): pragmatic depression assessment in the general population. Psychiatry Research, 186(1), 128-132. DOI: https://doi.org/10.1016/j. psychres.2010.08.018

Vandelanotte, C., Spathonis, K., Eakin, E., \& Owen, N. (2007). Website-delivered physical activity interventions a review of the literature. Am J Prev Med, 33(1), 54-64. DOI: https://doi.org/10.1016/j. amepre.2007.02.041

Van Dijk, T. K., Dijkshoorn, H., van Dijk, A., Cremer, S., \& Agyemang, C. (2013). Multidimensional health locus of control and depressive symptoms in the multi-ethnic population of the Netherlands. Social Psychiatry and Psychiatric Epidemiology, 48(12), 1931-1939. DOI: https://doi.org/10.1007/ s00127-013-0678-y

Webb, T. L., Joseph, J., Yardley, L., \& Michie, S. (2010). Using the Internet to promote health behavior change: A systematic review and meta-analysis of the impact of theoretical basis, use of behavior change techniques, and mode of delivery on efficacy. J Med Internet Res, 12(1), e4. DOI: https://doi. org/10.2196/jmir.1376

World Health Organization. (2014). World Health Statistics 2014. Geneva, Switzerland: World Health Organization. ISBN: 978-92-4-156471-7. Retrieved from: http://apps.who.int/iris/bitstr eam/10665/112738/1/9789240692671_eng.pdf.

How to cite this article: Alley, S., Andersson, M., Burton, N., Vandelanotte, C., Duncan, M., Happell, B., Short, C. and Rebar, A. (2017). Web-Based Intervention Preferences and Physical Activity Motivation of People with Depressive Symptoms. Health Psychology Bulletin, 1(1), 7-14, DOl: https://doi.org/10.5334/hpb.3

Submitted: 06 April 2017 Accepted: 09 July 2017 Published: 30 August 2017

Copyright: ๑ 2017 The Author(s). This is an open-access article distributed under the terms of the Creative Commons Attribution 4.0 International License (CC-BY 4.0), which permits unrestricted use, distribution, and reproduction in any medium provided the original author and source are credited. See http://creativecommons.org/licenses/by/4.0/.

$\mathrm{u}[\quad$ Health Psychology Bulletin is a peer-reviewed open access journal published by Ubiquity Press. 